\title{
Disseminated Low Grade Glioma in Children and Young Adults
}

\author{
R. Bell, A.A. Kirkwood, D. Hargrave, A. Michalski, H. Hyare, T. Jacques, S. Stoneham, Y. \\ Chang, N. Fersht, M. N. Gaze, K. Phipps, A. Shankar* \\ Consultant in Paediatric \& Adolescent Oncology, University College London Hospitals NHS Foundation Trust \\ London \\ *Corresponding Author: A. Shankar, Consultant in Paediatric \& Adolescent Oncology, University \\ College London Hospitals NHS Foundation Trust London
}

\begin{abstract}
Background: Disseminated low-grade gliomas [d-LGG] in children, teenagers and young adults [TYA] are rare and the treatment outcome is worse than for than for those with localised disease. We present a retrospective institutional and ethical review board approved report of $d-L G G$ in children and TYA treated at the London Cancer Paediatric \& Adolescent Neuro-Oncology Service.
\end{abstract}

Purpose: Between 1998 and 2014, 36 patients with d-LGG either at diagnosis or during follow up were identified. Anonymized data were retrospectively collected from the hospital databases and included patient demographics, modality of diagnosis, treatment received, recurrences or disease progression and outcome.

Results: The median age of our cohort was 4.5 years and median follow up was 6 years. $60 \%$ had disseminated disease at diagnosis; pilocytic astrocytoma was the commonest histological subtype $(n=18$, 50\%). Chemotherapy was the most common treatment modality utilised. The 6-year overall survival for those with localised disease with dissemination during follow-up and disseminated disease at presentation were $77.9 \%$ and $76.1 \%$ respectively. Progression free survival at 1, 2 and 3 years were 64.3\%, 50\% and $21.4 \%$ and $57.3,43 \%$ and $38.2 \%$ respectively.

Conclusion: Children with d-LGG treated have a poor outcome. A significant proportion of patients have multiple recurrences or disease progressions. There appears to be no difference in OS in those who had disseminated disease at diagnosis compared to those who developed disseminated disease at a later time point during follow up. Prospective international studies with molecular genetic profiling will help clarify the best treatment approach for this group of patients.

\section{INTRODUCTION}

Low-grade gliomas [LGG] and in particular, pilocytic astrocytomas, are the most common of childhood tumours of the central nervous system [Bergthold et al 2014 [1], Gupta et al 2017 [2], Ryall et al 2017 [3]]. These are a mixed group of tumours with a World Heath Organization grading of I or II with a generally good outcome [Ryall, et.al, 2017 [3]. Leptomeningeal dissemination [spread via the cerebrospinal fluid [CSF] pathways] is rare with reported incidence of around $5 \%$ at diagnosis to $10 \%$ at progression [Chamidine et al 2016 [4], Dodgshun et al, 2016 [5], Yecies et al 2018 [6]] Hence, published data is limited on the incidence, natural history, patterns of dissemination or clinical outcome in both children and TYA with disseminated LGG [d-LGG]. While some reports suggest that children with d-LGG have an acceptable treatment outcome [Tsang et al 2017 [7], Chamidine et al 2016 [4], Bian et al 2013 [8],
Perilongo et al 2003 [9], Hukin et al 2002], a few other published series depict a more dismal outlook [Von Hornstein et al 2011 [11], Rodriguez et al 2012 [12]]. As the natural history of the disease remains uncertain, it is unsurprising that there is also ambiguity with regard to the most effective treatment for children with d-LGG [Chamidine et al 2016 [4], Gnekov et al 2004 [13], Akar et al 2000 [14]]. In this report, we seek to initiate a dialogue on how this group of patients can best be managed using retrospectively gathered information on treatment outcomes in thirty-six children and adolescents with disseminated disease treated at the London Cancer Paediatric and Adolescent Neuro-Oncology Service [University College and Great Ormond street Hospitals, North London Cancer Network] UK.

\section{Patients And Methods}

We retrospectively reviewed the records of children and adolescents with a diagnosis of 
low-grade glioma [LGG] treated at The London Cancer Paediatric and Adolescent NeuroOncology Service [two foundation trust NHS hospitals; University College London Hospitals and Great Ormond Street Hospital for Children working as a joint single centre at 2 sites] between $1^{\text {st }}$ March 1998 and 23 ${ }^{\text {rd }}$ September 2014. The criterion for a diagnosis of LGG was based on the World Health Organisation classification of brain tumours [Louis et al 2016]. The two main inclusion criteria were: age up to $25^{\text {th }}$ birthday, presence of disseminated disease either at diagnosis or at a later time point during follows up. Tissue diagnosis was not mandatory if the radiological diagnosis was unequivocal of LGG; especially when the primary tumour was located in the optic pathways, hypothalamic chiasmatic [HC] region and dorsal mid brain region [tectum] or if the patient had neurofibromatosis type 1 [NF1] and the primary tumour was located in the optic pathways or $\mathrm{HC}$ region.

\subsection{Data Collection}

Anonymized patient data [basic demographic information, treatment administered and treatment response] were retrospectively collected from the hospital databases at our joint centre. Clinical parameters included: patient demographics (sex, age at diagnosis), date and modality of diagnosis (i.e. histological or radiological), tumour location, histological subtype of LGG [if tumour was biopsied or resected] and molecular mutations (where available) of the primary tumour. We looked specifically at when dissemination occurred (i.e. at diagnosis, progression or at recurrence), the type and duration of treatment received, recurrences or disease progression and current patient status. Consent for treatment, including collection of anonymized patient data, was obtained according to the prevailing institutional and ethical committee guidelines of the joint centre. Additionally, approval for transfer of data between the two hospitals was secured from the ethical committees of both hospitals. Any irregularities in information were corrected after discussion with the appropriate clinicians and hospital data managers. The institutional review boards at both Great Ormond Street Hospital for Children NHS Foundation Trust and the University College London Hospitals NHS Foundation Trust approved this retrospective review.

\subsection{Staging Procedures Including Imaging}

\subsubsection{Imaging}

The standard neuro-oncology practice for imaging in all patients was conventional structural magnetic resonance imaging (MRI) of brain and spine with a standardized brain tumour MRI protocol consisting of the following sequences: 3-dimensional (3-D) T1weighted (T1W), axial Fluid-Attenuated Inversion Recovery (FLAIR), axial T2-weighted (T2W), axial Diffusion Weighted Imaging (DWI), and 3-D contrast-enhanced T1W, performed on a 1.5 and/or 3 tesla MR system

\subsubsection{Tumour Location and Dissemination}

Patients were categorized as having localised or disseminated LGG at first diagnosis or during follow up. Primary tumour sites were based according to their location seen on magnetic resonance imaging $[\mathrm{MRI}]$ i.e. supratentorial, infratentorial or spinal.

\subsubsection{Diagnostic Criteria}

Diagnosis of LGG was either based on histology and or radiology. Radiological diagnosis was based on the findings of typical MRI features associated with LGG and included homogenous T2W signal intensity, well-defined tumour margins and minimal contrast enhancement. In a subset of patients, a first diagnosis of LGG was determined radiologically but a subsequent histological diagnosis confirmed LGG when the patient had surgery at disease progression or recurrence. Cerebrospinal fluid analysis [CSF] was not performed routinely at diagnosis or during follow up surveillance

\subsubsection{Treatment}

Treatment modalities were observation alone, chemotherapy, radiotherapy, surgery or any combination of the latter 3 treatment modalities. If surgery was the main treatment modality; the extent of surgical resection was further defined and categorised as a] complete resection, b] gross macroscopic resection [less than total resection] and debulking surgery [less then gross macroscopic resection]. Radiotherapy: This was either focal to the primary and/or cranio-spinal radiotherapy. A diverse number of chemotherapy regimens were used in this cohort and some patients also received bevacizumab, a monoclonal antibody that blocks angiogenesis by inhibiting vascular endothelial growth factor.

\subsection{Response Criteria}

Standard cross sectional MRI was the imaging modality used to assess treatment response. The response assessment standards were according to the RANO [Response Assessment in NeuroOncology] standards for LGG [van den Bent et al 2011] i.e. complete response [CR], partial 
response $[\mathrm{PR}]$, stable disease and progressive disease. The usual time point for initial treatment response MRI assessment was 6-8 weeks after completion of radiation therapy. Occasionally, serial imaging assessments were performed when pseudo-progression was suspected with retrospective determination of imaging changes, in combination with clinical status and corticosteroid use.

\subsubsection{Follow up}

The first follow-up visit was usually around 6-8 weeks after completion of treatment, followed by clinical evaluations at 3 to 4 monthly intervals during the first year after treatment and at 4-6 monthly intervals during the second and third years after treatment. Interval imaging during follow-up surveillance was usually between 4 - 6 months.

\subsection{Statistical Analyses}

Descriptive statistics have been used with Kaplan-Meier survival analyses used for all time-to-event endpoints. Progression free and overall survival (PFS and OS) times are calculated as the time between the date of diagnosis and the date of the first progression,

Table 1: Baseline characteristics of LGG patients relapse or death (whichever came first) for PFS or death for OS. Patients without an event were censored at the date last seen. PFS2 and OS2 are defined as the time from the first progression or relapse until the second event (progression, relapse or death of PFS2 or death for OS2) with patients who do not experience a second event censored at the date last seen. Only patients who experienced a first progression or relapse were included in PFS2 and OS2 analyses. Cox regression was used to assess associations between age and histology and overall survival. All analyses were performed using STATA version 15.1 (STATA corp, Texas)

\section{RESULTS}

\subsection{Demographics}

A total of 36 patients are included in this retrospective report. The median age of the cohort was 4.5 years [range: 6 months-20 years]. Eight $(22 \%)$ patients also had neurofibromatosis type 1 [NF1] whilst 1 patient (3\%) had tuberous sclerosis. The demographics and disease characteristics at diagnosis of these 36 patients are shown in table 1 .

\begin{tabular}{|c|c|}
\hline Characteristic & \\
\hline Age, median (range) & 4.5 years $(0.5-20)$ \\
\hline Sex, N (\%) & \\
\hline \begin{tabular}{l|l} 
Male \\
\end{tabular} & $18(50)$ \\
\hline Female & $18(50)$ \\
\hline Primary site, N (\%) & \\
\hline \begin{tabular}{l|l} 
Cerebellum \\
\end{tabular} & $11(30.6)$ \\
\hline Cerebral hemisphere & $2(5.6)$ \\
\hline Interventricular & $2(5.6)$ \\
\hline Optic nerve & $8(22.2)$ \\
\hline Spinal cord & $5(13.9)$ \\
\hline Thalamus & $3(8.3)$ \\
\hline Brainstem & $1(2.8)$ \\
\hline Suprasellar & $1(2.8)$ \\
\hline Tectal plate & $2(5.6)$ \\
\hline Missing & $1(2.8)$ \\
\hline Diagnosis type, $\mathbf{N}(\%)$ & \\
\hline Histology & $23(63.9)$ \\
\hline Radiology (with NF1 diagnosis) & $4(11.1)$ \\
\hline Histology and radiology (with NF1 diagnosis) & $4(11.1)$ \\
\hline Radiology without NF1 diagnosis & $5(13.9)$ \\
\hline Histology, N (\%) & \\
\hline $\begin{array}{ll}\text { Pilocytic } \\
\end{array}$ & $18(50.0)$ \\
\hline Low grade & $17(47.2)$ \\
\hline Pilomyxoid & $1(2.8)$ \\
\hline
\end{tabular}




\begin{tabular}{|c|c|}
\hline Molecular mutations, N (\%) & \\
\hline BRAF V600E mutations & $1(2.8)$ \\
\hline BRAF translocations i.e. BRAF-KIAA1549 & $5(13.9)$ \\
\hline No mutation documented & $3(8.3)$ \\
\hline Not done & $26(72.2)$ \\
\hline Other: non-braf malignant mutation & $1(2.8)$ \\
\hline \multicolumn{2}{|l|}{ Localised or disseminated at diagnosis, N (\%) } \\
\hline Localised & $14(38.9)$ \\
\hline Disseminated & $22(61.1)$ \\
\hline
\end{tabular}

\subsubsection{Mode of Diagnosis}

All 36 patients had histological confirmation of a low-grade glioma [LGG]: either at diagnosis $(27 / 36,75 \%)$; or at recurrence/ progression $(9 / 36,25 \%)$. In the latter group, the initial LGG diagnosis was based on the combination of clinical findings and typical MRI appearances, with 4 in the context of associated NF1. Pilocytic astrocytoma [PA] was the commonest histological type of LGG seen in our cohort [table 1]

\subsection{Primary Tumour Location}

Location of the primary tumours is shown in table 1. The most common primary site was the cerebellum $(n=11,31 \%)$, followed by the optic pathway/ hypothalamic chiasmal region $1(n=8$, $22 \%)$ and spinal cord $(\mathrm{n}=5,14 \%)$ respectively.

\subsubsection{Molecular mutations}

Testing for molecular alterations was performed in ten patients $(28 \%)$. Of these ten patients, 5 had the KIAA1549-BRAFfusions, 1 had the BRAF V600E insertion mutation and 1 had a
non-BRAF mutation. No molecular genetic alterations were identified in the remaining 3 patients.

\subsection{Dissemination of LGG - Time Points}

Twenty-one patients (58\%) had disseminated LGG at first presentation while the remaining 15 (42\%) patients developed dissemination at first progression.

Sites of disseminated disease at diagnosis were as follows: within the brain alone $[n=5]$, spine alone $[n=6]$ or dissemination throughout the neuraxis $[\mathrm{n}=10]$

\subsubsection{Treatment and outcome}

Sixteen patients had chemotherapy as part of their treatment program, with 13 receiving chemotherapy $[n=13 ; 36 \%]$ alone as their primary treatment. Table 2 shows the treatment modalities used our cohort of patients. The chemotherapy regimen used in all but one patient was vincristine and carboplatin.

Table 2: Treatment modalities

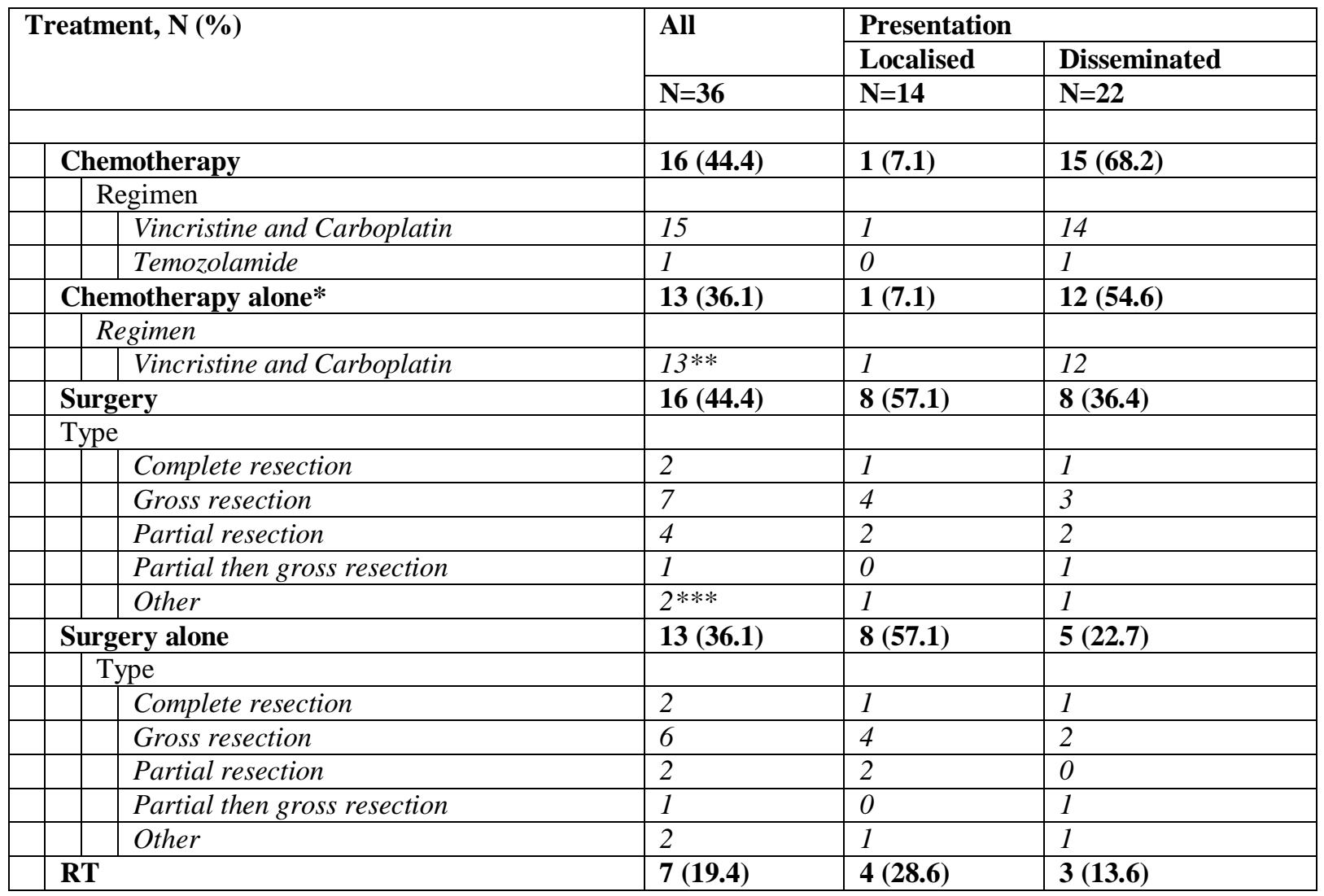




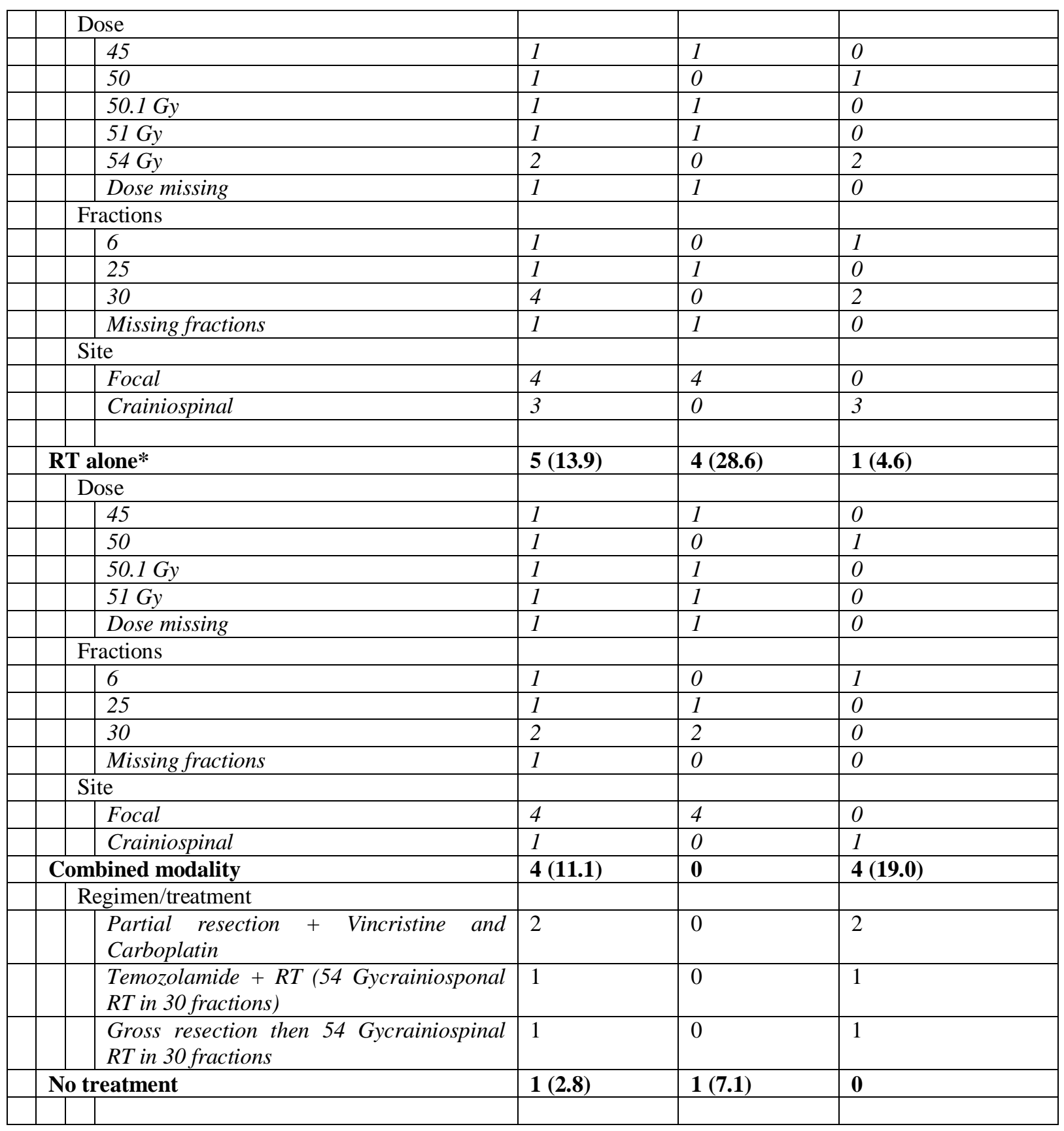

*Also biopsied (chemotherapy: $n=2, R T: n=1) * *$ One patient changed to Cisplatin- vincristine at week 25 due to carboplatin toxicity, $* * *$ Shunt to reduce pressure and a biopsy $(n=1)$, not specified $(n=1)$

Five patients received radiotherapy alone as their primary treatment, 4 patients with localised disease received focal radiotherapy while one patient who developed disseminated disease during surveillance received cranio-spinal radiotherapy. Radiotherapy doses to the primary were between 50-60Gy; 1.8Gy/ fraction while cranio-spinal radiotherapy doses ranged between 24-36Gy; 1.8Gy/ fraction. Radiotherapy had to be aborted in the patient with disseminated disease after 6 fractions due to overwhelming sepsis. Combined modality treatment: 5 patients $(17 \%)$ received combined modality treatment. Surgery: Thirteen patients had surgery alone as the primary treatment. Only two patients achieved complete primary tumour resection while the remaining had macroscopic residual disease after surgery. The degree of tumour resection was unclear in 1 patient.

\subsubsection{Treatment Response}

Details of treatment response were available in 34 of the thirty-six patients [one patient died of sepsis during the second week of treatment and response outcome was not available in the other patient]. Twenty-one patients had disease progression during first line treatment, 12 achieved a PR and 1 patient achieved CR. The only patient who achieved a CR had surgery for non-disseminated disease at diagnosis. 
Response according to primary treatment was as follows: surgery [ $\mathrm{n}=13$; CR-1; PR-6; PROG-6], chemotherapy alone [n=13; CR-0; PR-3; PROG10], radiotherapy alone [ $\mathrm{n}=5$; PR-1; PROG-3, death due to sepsis -1], combined modality treatment [n=5; CR-0; PR-2; PROG-2, NA=1]. The six patients who progressed after surgery had residual disease and progressed soon after surgery

\subsection{Median Follow-Up}

The median follow-up is 6.0 years (range: 10 days -13 years)

\subsubsection{Localized disease at presentation}

The median time to progression/relapse was 1.7 years (range: 17 days -10.3 years). The PFS ratesat 1,2 and 3 years were $64.3 \%$ [95\% CI:
$34.3-83.3$ ], $50 \%$ [22.9-72.2] and $21.4 \%$ [5.2$44.8]$ respectively.

\subsubsection{Disseminated disease}

Of the twenty-two patients diagnosed with disseminated disease at first presentation, 16 patients had subsequent disease progression and 5 have died [2 without progression] giving a total of 18 PFS events. The median PFS time was 1.7 years (range, for those with an event: 10 days -5.3 years). The PFS rates at 1,2 and 3 years were 57.3 [33.9-75], 43\% [22.9-62.4] and 38.2 [18.4-57.9] respectively.

PFS rates for those with localised disease and disseminated disease at presentation are shown in figure 1

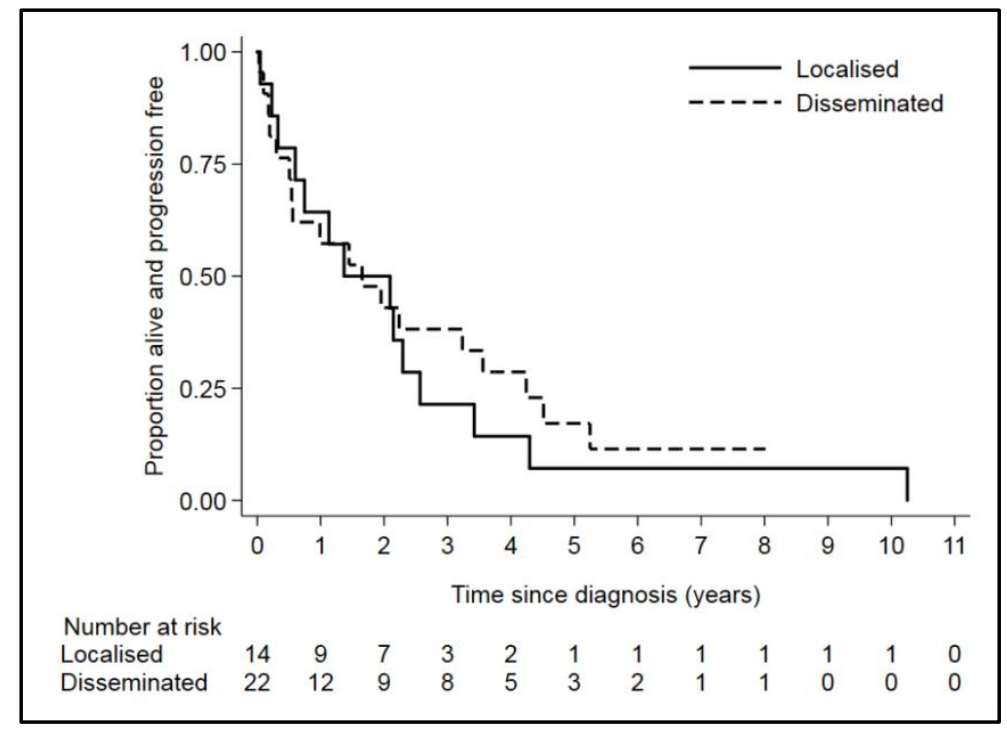

Figure1: Progression Free survival

\subsection{Second Progression}

Seventeen patients have had two or more progressions. The median follow up [censored at death] is 1.6 years from first progression. See figure 2

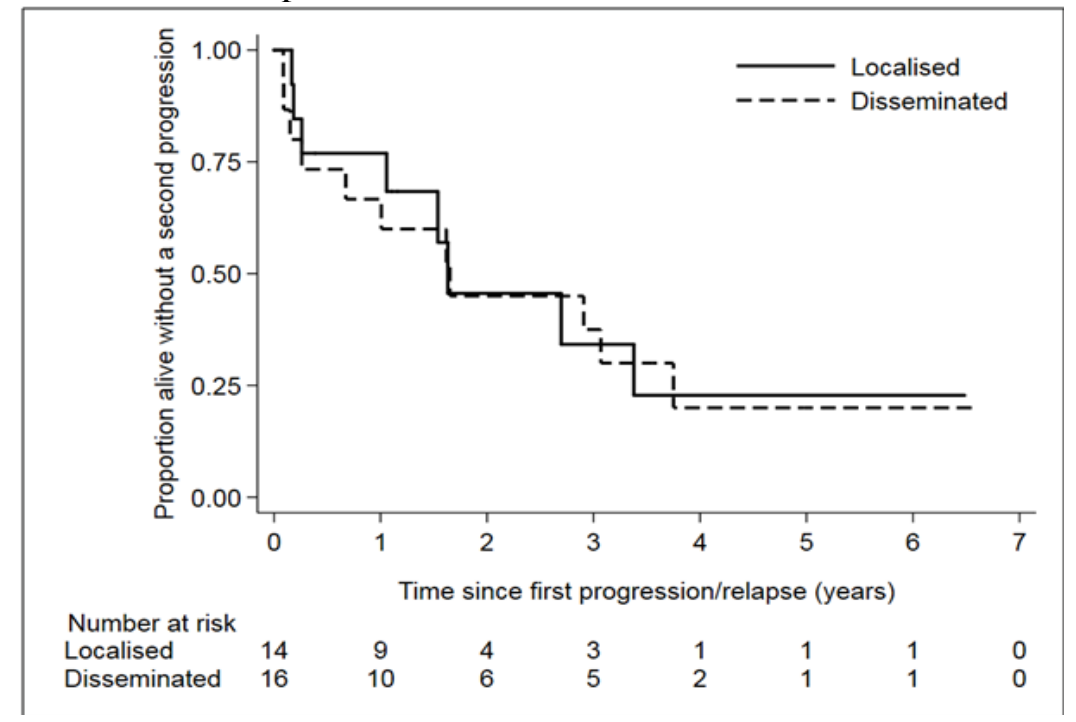

Figure2: PFS from first progression 


\subsubsection{Localised disease at presentation}

Eight patients have had a PFS event after progression to disseminated disease [6 progressions and 2 deaths with no second progression]. The median PFS2 after dissemination was 1.6 years [range, patients with an event: 61 days -3.8 years]. The 2 deaths occurred early $[61$ and 67 days post dissemination]. The PFS 2 rates at 1 and 2 years were 76.9\% [44.2-91.9] and 45.6\% [37.5-84.6] respectively.

\subsubsection{Disseminated Disease at presentation}

Eleven patients have had a second progression [11 PFS events; no deaths between PFS1 and PFS2]. The median PFS2 time after dissemination was 1.7 years [range, 32 days -3.8 years] The PFS2 rates at 1 and 2 years were $66.7 \%$ [37.584.6] and 45.0\% [19.4 -67.8] respectively.

\subsection{Overall Survival}

\subsubsection{Localized disease at presentation}

Four $(27 \%)$ patients have died, 2 of progressive disease and 2 due to an intra cranial bleed and one related to treatment related complications [post anaesthetic complication]. The OS rate at 5 years and at the median follow-up of 6 years was $77.9 \%(45.9-92.3)$.

\subsubsection{Disseminated disease at presentation}

Five $(24 \%)$ patients have died, 4 from progressive disease [including one within 10 days of diagnosis] and 1 due to treatment related complication [respiratory arrest due to septicaemia]. The OS rate at 5 and 6 years was $76.1 \%(51.6-89.3)$.

Overall survival for those with localised disease and disseminated disease at presentation are shown in figure 3

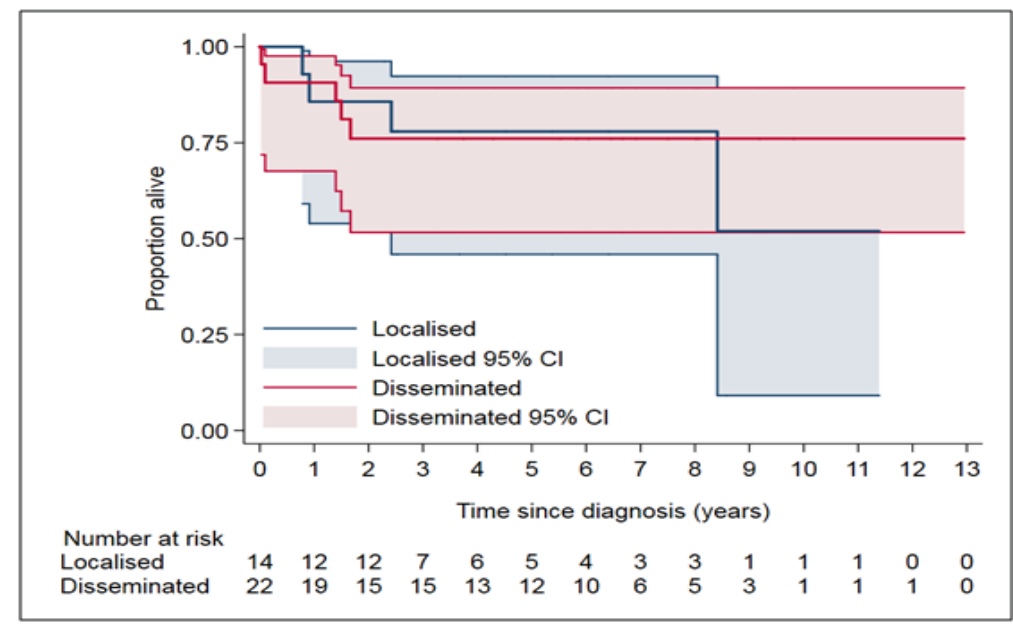

Figure3: Overall survival

\subsection{Overall Survival from Last Progression}

Figure 4 shows the OS from last reported progression. The OS rate at 1 year for patients with localised disease was $64.8 \%$ [31-85.2] and for those with disseminated disease was $69.8 \%$ [31.8-89.4]. Six patients have a follow up $>3$ years and 3 patients have a follow up period of $>4$ years. Figure XII shows the swimmer plots showing all events Figure 5 shows a swimmer plot for all events including death

Figure 6 shows the OS by age group.

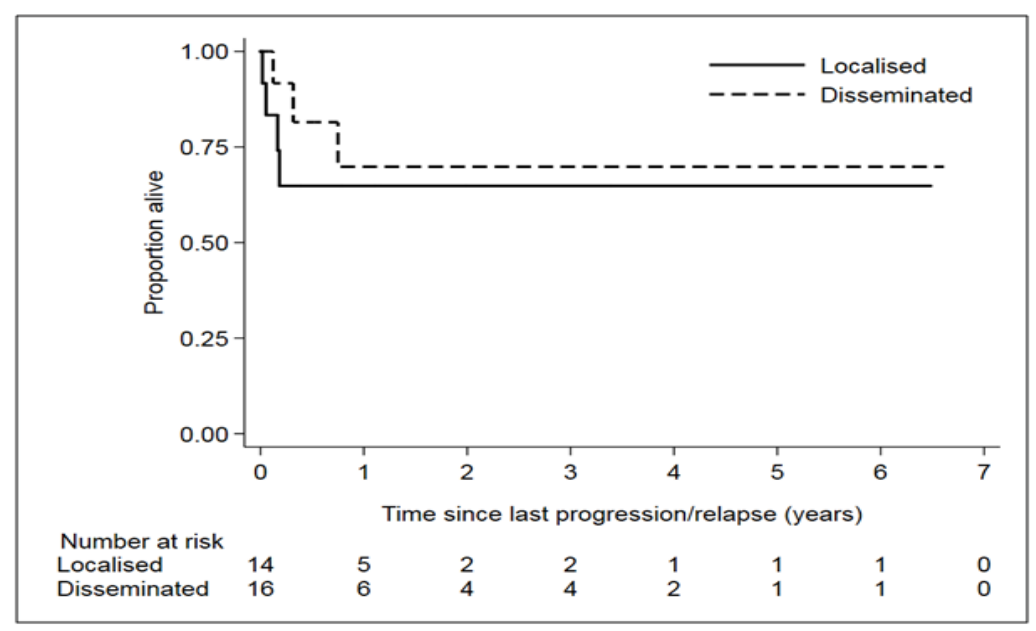

Figure4: Overall Survival from last reported progression 


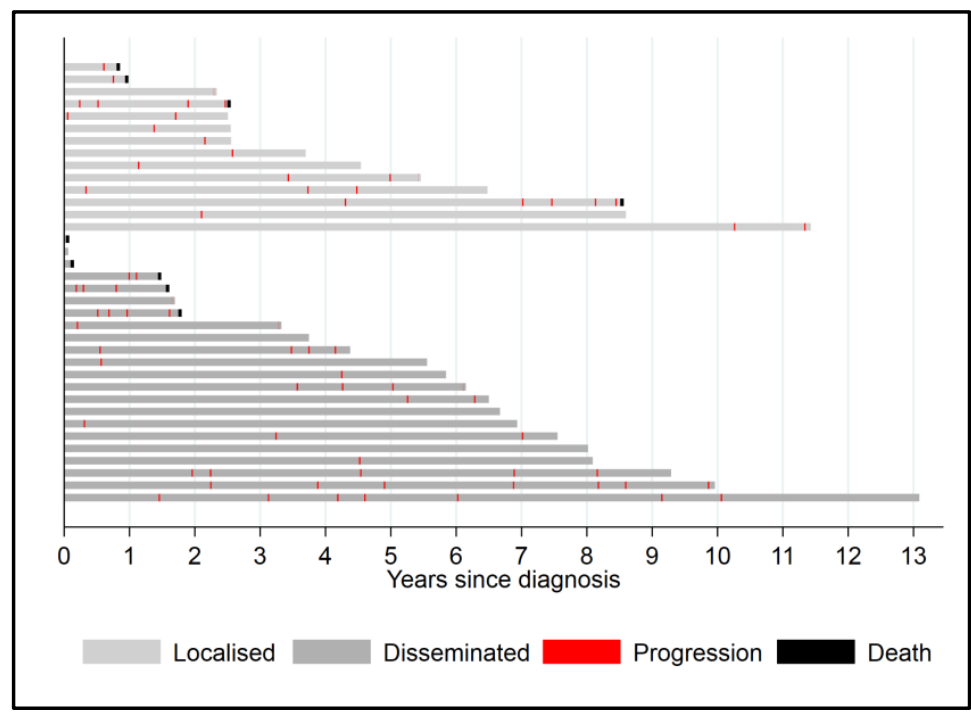

Figure5: Swimmer plots showing all events

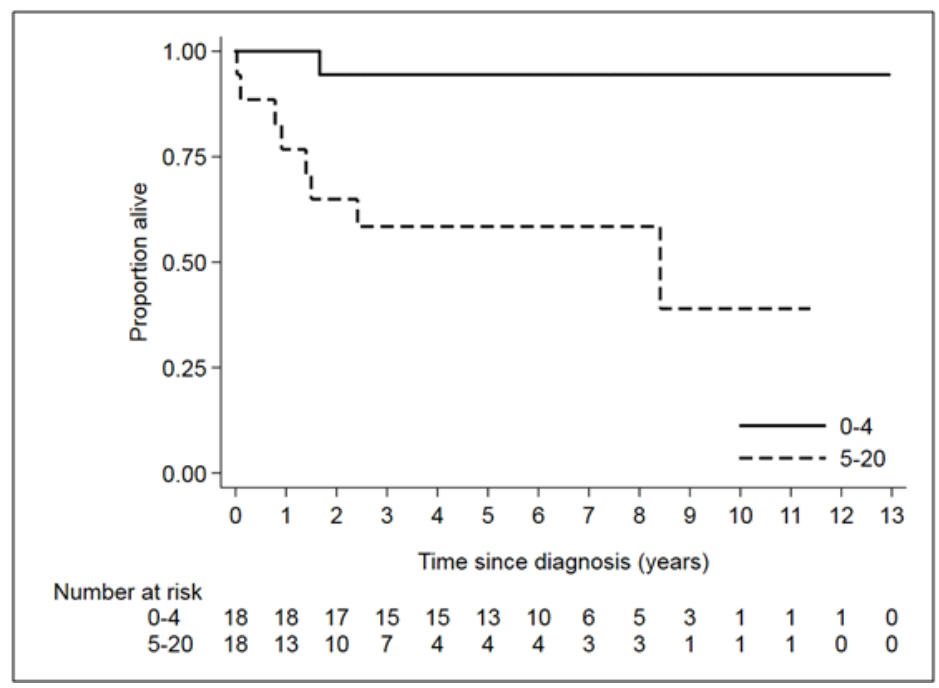

Figure6: Overall survival by age group

\subsection{Risk Factors for Poorer Overall Survival}

Although there was no difference seen between pilocytic astrocytomas and non-pilocytic lowgrade gliomahistologies, older patients appear to be at high risk of death with a 13-fold increase for each log increase in age. Splitting age at the median [figure 6] suggested that the older group had a more than 11 fold increase in risk with $1 / 18$ dying in the 0-4 age group compared to $8 / 18$ in 5+ age group (HR (stratified by localised/disseminated disease at diagnosis) 11.67 (95\% CI: $1.36-100.16), p=0.025$ ).

\section{DISCUSSION}

Disseminated LGG in children is rare and consequently, published reports on the natural history, treatment strategy and treatment outcomes are limited [Hukin et al 2002 [10], von Hornstein et al 2011 [11], Chamidine [4] al 2016, Tsang [7] et al 2017]. While this retrospective report, represents one of the larger cohorts [St Jude Children's Hospital - n=38; Dana Farber and Melbourne children's Hospital - $n=10$, German multi centre report by von Hornstein [11] et al - $n=61$ ] of children and adolescents with disseminated LGG, the number of patients in this cohort is still small.

Of the 27 patients, in whom the diagnosis of LGG was based on histological evidence at presentation, pilocytic astrocytoma $[\mathrm{n}=18]$ was the commonest histological subtype. This is in consonance with the German HIT-1996 trial $[\mathrm{n}=17 / 22]$ as well as the recent report from St. Jude Children's Hospital [ $\mathrm{n}=23 / 38$ ] [Gnekov [15] et al 2012, Chamidine [4] et al 2016]. Both reports included patients with primary and secondarily disseminated LGG and the St. Jude series in particular has excellent long-term follow up. The two most common sites of primary tumour in our group were the cerebellum $[n=11]$ and the optic chiasm $[n=8]$. It has been previously reported that patients with 
optic chiasmal LGG and midline tumours have a relatively higher risk of developing disseminated disease and poorer treatment outcome [Pollack et al 1994 [16], Prados et al 1994 [17], von Hornstein et al 2011 [11]]. The reasons advanced for a worse outcome for tumours at these sites is the difficulty in obtaining optimal maximal safe resection of tumours, in particular when tumoural extension goes into the hypothalamus \& ventricle and has a very wide and continuous rate of growth [Alvord 1988 [18]].Published literature confirms that maximal safe surgical resection is crucial not only on the duration of survival but also to maximize quality of life in patients with localized LGG [Clark 2019 [19]]. Neuroplasticity in children should allow cerebral remapping over time and therefore second look surgery can be reviewed at a later time to achieve maximal resection. It is likely this equally applies to those with disseminated disease [Tsang et al 2017 [7]].

Molecular tumour biology was only analyzed in 10 patients [28\%], of whom 5 had the KIAABRAF mutation and 1 the BRAF v600E mutation [table 1]. All 6 with BRAFF molecular alterations were alive at the LFU visit. It has been shown that LGG patients with BRAF fusions have an improved survival outcome [Hawkins C et al 2011 [20]]. It is possible that some patients in our cohort had a higher-grade tumour than shown by histology; as it is now evident that histology alone is inadequate for the diagnosis of brain tumours. Tumours that harbor the K27M histone H3 mutation are commonly seen in midline structures such as the pons or the thalamus and are considered high-grade tumourswith a poor outcome. However this mutation can also be seen in $10 \%$ of low-grade midline tumours [Ryall S et al 2017 [3]]. In adults with embryonal tumours, acombined approach based on clinical, radiological, histological and molecular genetic information has been used to accurately distinguish astrocytoma subtypes and inform and tailor effective therapy [Halliday et al 2018 [21]].

Children in whom safe maximal resection can be achieved have an excellent overall (OS) and event-free survival (EFS) and often, do not require adjuvant therapy. However, children with unresectable tumours or those with disseminated disease often experience multiple progressions and require additional treatment. It is of interest to note that those who received chemotherapy alone $[n=13]$ as their initial primary treatment have had a relatively good treatment outcome with11 [77\% \} alive at their LFU visit $[1$ had localised disease at diagnosis; 10 had disseminated disease at diagnosis, all of these 10 have survived at least 3 years from diagnosis, with 7 more than 6 years and 3 more 9 years]. Chemotherapy was the preferred treatment modality in our small cohort primarily to avoid or delay radiotherapy as many of our children were very young. This approach is similar to the practise adopted by other cooperative groups in hope of preventing some of the more late adverse effects of radiotherapy. Additionally, the advantages of this treatment modality include its tolerable side effects and the reduced requirement of inpatient hospital stay in most patients. A report from St Jude Children's Research Hospital [Tsang [7] et al 2017], suggests that children with disseminated LGG treated with cranio-spinal radiotherapy experienced a longer EFS than those treated with chemotherapy alone and with similar OS rates. We are unable to confirm this observation in our small cohort as only 6 patients has radiotherapy as their front line treatment. The combination of vincristine and carboplatin was the most common chemotherapy regimen used in our cohort and observed treatment response was similar in both primary and disseminated sites.

Malignant transformation was not seen in any of our patients and this is perhaps not surprising as malignant transformation of LGGs in children is reportedly a very rare phenomenon [Chamdine et al 2016 [4]]. Whilea high proportion of adults with WHO grade 2 infiltrative astrocytoma experience malignant transformation, the longterm risk of transformation in histological identical neoplasms in children is less than $10 \%$ [Broniscer A et al 2007 [22]]. It is possible that the lack of histological confirmation of transformation at autopsy in patients who died of progressive LGG may have caused underestimation of malignant transformation. There was no difference in the PFS rates between those who had d-LGG at presentation versus those who had initially localized disease but with subsequent dissemination [Figure 1].

Disseminated disease at diagnosis was present in just over half of our patient cohort [ $\mathrm{n}=21]$. While 15 patients subsequently developed disseminated disease, the median time to progression was 2.1 years, which is longer that reported by the St Jude's group [11 months]. We however, did not find any difference in OS dueprimary disseminated LGG vs. secondarily 
disseminated disease or according to the histological subtypes, though there appeared to be a difference according to age with an increase in risk in older patients. However, due to the small numbers of patients and events and non-uniform treatment it is difficult to draw any firm conclusions about whether an older age actually confers a worse prognosis.

\subsection{Survival Outcomes}

The 5-year overall survival outcome of those who had disseminated disease at presentation [ $\mathrm{n}=22]$ was [75.2\%; 95\% CI 50.3-88.9]. This is broadly similar to recent published reports in literature of childhood disseminated low-grade glioma [Chamidine et al 2016 [4], Tsang DS et al 2017 [7]] but decidedly superior to the results reported by Hukin et al [Hukin et al 2003 [23]]. This probably is due the close clinical and radiological surveillance of children with LGG where early progression/ recurrence is detected in a timely manner with institution of appropriate treatment rapidly. It is however also possible that with longer follow up, the survival rates of our cohort could fall further. Interestingly in our series, the overall survival outcome for those who had disseminated disease at diagnosis was similar to those who initially had localised disease but subsequently developed disseminated disease [75.2 vs. $78.9 \%$ ].

The optimal management of children with disseminated LGG should be to limit the risk of treatment-related toxicity without compromising disease control. Consequently, increasing effort should be directed to retaining the demonstrated efficacy of chemotherapy in maximizing the cure and avoid or defer radiation treatment. However, there is no consensus on the most effective treatment modality. In our small series, it is difficult to determine the most efficacious treatment modality due to the small numbers who received the various treatment programmes. With the advances in genomic sequencing elucidating the molecular genetic spectrum of low-grade gliomas, the evolution of advanced functional MR imaging and the use of selective mutant drug targets with emerging small molecules, it is possible that there will be a paradigm shift in the management of disseminated disease in the future.

However for the present, the recognition of the efficacy of chemotherapy and the data on the adverse late effects of radiation, and it is likely that chemotherapy will be the preferred first line treatment modality in disseminated childhood
LGG. While platinum based chemotherapy regimens are currently the most preferred chemotherapy regimens with good response rates [Mora $\mathrm{J}$ et al 2018 [24]], single agent vinorelbine [Cappellano AM et al 2015 [25]] and vinblastine [Bouffet et al 2012 [26]] also show promising response with a low toxicity profile and providing a good quality of life for patients

\subsection{Limitations}

A major limitation of this study is the fact that this report is retrospective in nature and the patient numbers are small.

Secondly, molecular profiling was only done in less than a third of the whole cohort [N=10]. It is now well known that histological appearances can be deceptive i.e. phenotypically similar LGG can have a different genetic profile underlying a different biology

Thirdly, a number of upfront treatment programs have been used and it is difficult to commend one over the other due to small patient numbers

\subsection{Future Directions}

We have shown in this report that children with disseminated low-grade glioma have an inferior outcome. The past decade has seen tremendous development in the recognition of the molecular biology underlying pediatric gliomas powering optimism to achieve effective treatment options. Disseminated pilocytic astrocytomas show genetic features similar to classic pilocytic astrocytoma, including a similar incidence of KIAA1549-BRAF fusions, BRAF mutations and a stable genetic profile. Given common activation of the signal transduction pathways such as MAPK, the use of specific inhibitors may be postulated for the treatment of disseminated pilocytic astrocytomas, along with standard chemo- and/or radiotherapy. What is needed is large scale international collaborative studies on the biologic subgroup of patients with disseminated low-grade gliomas to characterize distinct molecular genetic profile, advanced functional imaging and clinical features, with clear relevance for patient management. This may help reframe the poor prognosis that our historical patient group experienced and allow us to offer more tailored therapy to those most at risk of recurrence

\section{ACKNOWLEDGEMENTS}

Dr M. N. Gaze and Dr H Hyare are supported by the National Institute for Health Research 
[NIHR], University College London Hospitals Biomedical Research Centre.

\section{REFERENCES}

[1] Craniospinal irradiation for treatment of metastatic paediatric low-grade glioma. J Neurooncology. 2017. 134:317-324. Tsang DS, Murphy ES, Ezell SE, Lucas JT Jr, Tinkle C, Merchant TE.

[2] Impact of chemotherapy on disseminated lowgrade glioma in children and adolescents: report from the HIT-LGG 1996 trial. von Hornstein S1, Kortmann RD, Pietsch T, Emser A, Warmuth-Metz M, Soerensen N, Straeter R, Graf N, Thieme B, Gnekow AK.Pediatr Blood Cancer.2011. 56:1046-54.

[3] Disseminated glioneuronal tumors occurring in childhood: treatment outcomes and BRAF alterations including V600E mutation. Dodgshun AJ, SantaCruz N, Hwang J, Ramkissoon SH, Malkin H, Bergthold G, Manley P, Chi S, MacGregor D, Goumnerova L, Sullivan M, Ligon K, Beroukhim R, Herrington B, Kieran MW, Hansford JR, Bandopadhayay P. J Neurooncol. 2016. 128: 293-302

[4] Molecular characterization of disseminated pilocytic astrocytomas. Gessi M, Engels AC, Lambert S, Rothämel $\mathrm{T}$, von Hornstein $\mathrm{S}$, Collins VP, Denkhaus D, Gnekow A, Pietsch T. Neuropatho 1Appl Neurobiol. 2016. 42:273-8

[5] Low-grade gliomas and leptomeningeal dissemination: a poorly understood phenomenon. Perilongo G, Garrè ML, Giangaspero F. Childs Nerv Syst. 2003. 9: 197-203

[6] A comprehensive review of paediatric lowgrade diffuse glioma: pathology, molecular genetics and treatment. Ryall S, Tabori U, Hawkins C. Brain TumorPathol. 2017. 34: 51-6 1

[7] Metastatic low grade gliomas in children: 20 years experience at St Jude Children's Research Hospital. Chamdine O, Broniscer A, Wu S, Gajjar A, Qaddoumi I. Pediatr Blood Cancer. 2016. 63: 62-70

[8] Long-term outcomes of primarily metastatic juvenile pilocytic astrocytoma in children.Yecies D, Fisher PG, Cheshier S, Edwards M, Grant G. J Neurosurg Pediatr. 2018. 21: 49-53.

[9] Radiation Therapy for Optic Pathway and Hypothalamic Low-Grade Gliomas in Children. Tsang DS, Murphy ES, Merchant TE. Int J Radiat Oncol Biol Phys. 2017. 99: 642-651

[10] A clinicopathologic study of diencephalic pediatric low-grade gliomas with BRAF V600 mutation. Ho CY, Mobley BC, GordishDressman H, VandenBussche CJ, Mason GE, Bornhorst M, Esbenshade AJ, Tehrani M, Orr BA, LaFrance DR, Devaney JM, Meltzer BW,
Hofherr SE, Burger PC, Packer RJ, Rodriguez FJ. Acta Neuropathol. 2015. 130: 575-85.

[11] Chiasmatic low-grade glioma presenting with sacral intradural spinal metastasis.Akar Z, Tanriover N, Kafadar AM, Gazioglu N, Oz B, Kuday C. Childs Nerv Syst. 2000. 16: 309-11

[12] Leptomeningeal dissemination in children with progressive low-grade neuroepithelial tumors. Hukin J, Siffert J, Velasquez L, Zagzag D, Allen J. Neuro Oncol. 2002. 4: 253-60

[13] Pediatric low-grade gliomas: next biologically driven steps. Jones DTW, Kieran MW, Bouffet E, Alexandrescu S, Bandopadhayay P, Bornhorst M, Ellison D, Fangusaro J, Fisher MJ, Foreman N, Fouladi M, Hargrave D, Hawkins C, Jabado N, Massimino M, Mueller S, Perilongo G, Schouten van Meeteren AYN, Tabori U, Warren K, Waanders AJ, Walker D, Weiss W, Witt O, Wright K, Zhu Y, Bowers DC, Pfister SM, Packer RJ. Neuro Oncol. 2018. 20: $160-173$

[14] Molecular characterization of disseminated pilocytic astrocytomas. Gessi M, Engels AC, Lambert S, Rothamel T, von Hornstein S, Collins VP, Denkhaus D, Gnekow A, Pietsch T. Neuropathl ApplNeuro BIOL. 2016. 42: 273-8

[15] Epidermal growth factor receptor gene amplification and expression in disseminated pediatric low-grade gliomas. Tabori U, Rienstein S, Dromi Y, Leider-Trejo L, Constantini S, Burstein Y, Dvir R, Amariglio $\mathrm{N}$, Toren A, Rechavi G, Izraeli S, Aviram A. J Neurosurg. 2005. 103(4 Suppl): 357-61

[16] Expression analysis of juvenile pilocytic astrocytomas by oligonucleotide microarray reveals two potential subgroups. Wong KK, Chang YM, Tsang YT, Perlaky L, Su J, Adesina A, et al. Cancer Research. 2005. 65:76-84

[17] World Health Organization Histological Classification of Tumours of the Central Nervous System. International Agency for Research on Cancer, France. Louis DN, Ohgaki H, Wiestler OD, Cavenee WK (2016)

[18] Pilocytic astrocytoma with leptomeningeal dissemination. Bian SX1, McAleer MF, Vats TS, Mahajan A, Grosshans DR. Childs nervous system. 2013. 29:441-50.

[19] Leptomeningeal dissemination in children with progressive low-grade neuroepithelial tumors. Hukin J1, Siffert J, Velasquez L, Zagzag D, Allen J.. Neuro-oncology. 2002. 4: 253-60

[20] Low grade chiasmatic-hypothalamic gliomacarboplatin and vincristin chemotherapy effectively defers radiotherapy within a comprehensive treatment strategy - report from the multicenter treatment study for children and adolescents with a low-grade glioma - HITLGG 1996 - of the Society of Pediatric Oncology and Hematology (GPOH). Gnekow 
AK, Kortmann RD, Pietsch T, Emser A. KlinischePadiatrie. 2004. 216:331-42

[21] Long-term follow-up of the multicenter, multidisciplinary treatment study HIT-LGG1996 for low-grade glioma in children and adolescents of the German Speaking Society of Pediatric Oncology and Hematology. Gnekow AK, Falkenstein F, von Hornstein S, Zwiener I, Berkefeld S, Bison B, Warmuth-Metz M, Driever PH, Soerensen N, Kortmann RD, Pietsch T, Faldum A.Neuro-oncology. 2012. 14:1265-84

[22] Response assessment in neuro-oncology (a report of the RANO group): assessment of outcome in trials of diffuse low-grade gliomas. van den Bent MJ, Wefel JS, Schiff D, Taphoorn MJ, Jaeckle K, Junck L, Armstrong T, Choucair A, Waldman AD, Gorlia T, Chamberlain M, Baumert BG, Vogelbaum MA, Macdonald DR, Reardon DA, Wen PY, Chang SM, Jacobs AH. Lancet Oncology. 2011. 12:583-93.

[23] Dissemination of low-grade intracranial astrocytomas in children. Pollack IF, Hurtt M, Pang D, Albright AL. Cancer.1994. 73:2869-78

[24] Metastasizing low-grade gliomas in children. Redefining an old disease. Prados M, Mamelak AN. Cancer. 1994. 73: 2671-3

[25] Outcome by patients' age, tumor site, and treatment. Alvord EC Jr, Lofton S.Gliomas of the optic nerve or chiasm. Journal of Neurosurrgery. 1988. 68:85-98

[26] Extent of Resection Versus Molecular Classification: What Matters When? Clark VE, Cahill DP.Neurosurgery clinics of North America. 2019. 30: 95-101

[27] BRAF-KIAA1549 fusion predicts better clinical outcome in pediatric low-grade astrocytoma. Hawkins C, Walker E, Mohamed N, Zhang C, Jacob K, Shirinian M, Alon N, Kahn D, Fried I, Scheinemann K, Tsangaris E, Dirks P, Tressler R, Bouffet E, Jabado N, Tabori U. Clinical cancer research. 2011. 17: 4790-8
[28] A comprehensive review of paediatric lowgrade diffuse glioma: pathology, molecular genetics and treatment.Ryall S, Tabori U, Hawkins C. Brain tumour pathology. 2017. 34: 51-61

[29] The case for DNA methylation based molecular profiling to improve diagnostic accuracy for central nervous system embryonal tumors (not otherwise specified) in adults. Halliday GC, Junckerstorff RC, Bentel JM, Miles A, Jones DTW, Hovestadt V, Capper D, Endersby R, Cole $\mathrm{CH}$, van Hagen T, Gottardo NG.. Journal of Clinical Neuroscience. 2018. 47: 163-67

[30] Clinical and molecular characteristics of malignant transformation of low-grade glioma in children. Broniscer A, Baker SJ, West AN, Fraser MM, Proko E, Kocak M, Dalton J, Zambetti GP, Ellison DW, Kun LE, Gajjar A, Gilbertson RJ, Fuller CE. Journal of Clinical Oncology. 2007. 25: 682-9

[31] Treatment of childhood astrocytomas with irinotecan and cisplatin. Mora J, Perez-Jaume S, Cruz O.Clinical and translational oncology. 2018. 20: 500-507

[32] A lower-dose, lower-toxicity cisplatinetoposide regimen for childhood progressive low-grade glioma. Massimino M, Spreafico F, Riva D, Biassoni V, Poggi G, Solero C, Gandola L, Genitori L, Modena P, Simonetti F, Potepan P, Casanova M, Meazza C, Clerici CA, Catania S, Sardi I, Giangaspero F.Journal of neuro-oncology. 2010. 100: 65-71

[33] Single agent vinorelbine in pediatric patients with progressive optic pathway glioma. Journal of neuro-oncology.Cappellano AM, Petrilli AS, da Silva NS, Silva FA, Paiva PM, Cavalheiro S, Bouffet E. 2015. 121: 405-12

[34] Phase II study of weekly vinblastine in recurrent or refractory pediatric low-grade glioma.Bouffet E, Jakacki R, Goldman S, Hargrave D, Hawkins C, Shroff M, Hukin J, Bartels U, Foreman N, Kellie S, Hilden J, Etzl M, Wilson B, Stephens D, Tabori U, Baruchel S.Journal of Clinical Oncology. 2012. 30: 135 8-63.

Citation: R. Bell, A.A. Kirkwood, A. Shankar, et.al, Disseminated Low Grade Glioma in Children and Young Adults. ARC Journal of Cancer Science. 2020; 6(1):07-18. DOI:doi.org/10.20431/2455-6009.0601002.

Copyright: (C) 2020 Authors. This is an open-access article distributed under the terms of the Creative Commons Attribution License, which permits unrestricted use, distribution, and reproduction in any medium, provided the original author and source are credited. 